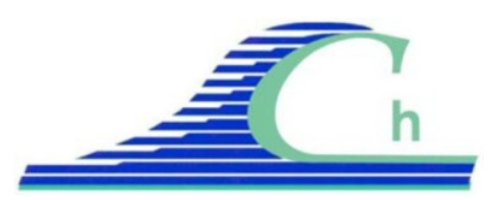

XII ${ }^{\text {èmes }}$ Journées Nationales Génie Côtier - Génie Civil

Cherbourg, 12-14 juin 2012

DOI:10.5150/jngcgc.2012.011-M @ Editions Paralia CFL

disponible en ligne - http://www.paralia.fr - available online

\title{
Interaction houle focalisée-courant dans une profondeur finie
}

\author{
Djalal MERKOUNE ${ }^{1}$, Julien TOUBOUL ${ }^{2}$, Nizar ABCHA ${ }^{1}$, \\ Dominique MOUAZE ${ }^{1}$, Alexander EZERSKY ${ }^{1}$
}

\author{
1 Laboratoire de Morphodynamique Continentale et Côtière UMR 6143 CNRS, \\ Caen, France. \\ djalal.merkoune@unicaen.fr \\ 2 Institut Méditerranéen d’Océanographie (MIO), UMR 7294 CNRS / IRD / AMU / \\ USTV, Toulon, France. \\ julien.touboul@univ-tln.fr
}

\section{Résumé :}

Nous étudions la propagation d'un paquet d'ondes à fréquence modulée dans une eau de profondeur finie en présence d'un courant. On a montré que la distance et l'amplitude de focalisation dépendent essentiellement du sens et de l'intensité de l'écoulement ainsi que de la fréquence d'excitation des ondes. Les résultats obtenus sont en bon accord avec ceux des simulations numériques.

Mots-clés : Houle focalisée - Houle-courant - Interaction

\section{Abstract:}

We study the propagation of a frequency modulated wave packet in finite depth water on the background of a hydrodynamic flow. It was shown that the distance and the amplitude of wave focusing depend on direction and value of the flow velocity and on wave frequency. The obtained results are in good agreement with numerical simulation.

Keywords: Wave focusing - Wave-current - Interaction

\section{Introduction}

Les vagues de grandes amplitudes, dites vagues scélérates, sont des vagues très complexes qui constituent un enjeu majeur pour la sécurité maritime. Leur apparition est observée lors des tempêtes d'une certaine importance, le plus souvent en plein océan dans les eaux profondes. Cependant on peut en rencontrer dans les zones côtières. Dans la littérature, de nombreux chercheurs ont tenté de comprendre la dynamique de ces vagues pour mieux maîtriser leur danger. Parmi les mécanismes susceptibles d'expliquer leur génération, la focalisation spatio-temporelle est souvent citée (KHARIF \& PELINOVSKY, 2003 ; PORUBOV et al., 2005). La compression d'un train d'ondes modulé en fréquence est conditionnée par la dispersion des ondes (dépendance de vitesse de groupe sur le nombre d'onde $\left(c_{g}=\partial \omega(k) / \partial k\right)$. En effet, des vagues courtes, qui se propagent moins vite que des vagues longues, seront rattrapées, puis dépassées par les vagues les plus longues dès qu'elles seront émises à l’avant du groupe. En général, ce mécanisme est bien décrit dans le cas de train d'ondes se propageant en profondeur 
infinie (KHARIF et al., 2001). Dans ces conditions de profondeur, TOUBOUL et al. (2007) ont étudié l'interaction du train d'ondes focalisant avec un courant uniforme. Ces derniers ont montré que si le courant transformait le point de focalisation en une zone plus étendue, la non linéarité, dans des cas de contre courant, compensait cet effet, et maintenait l'amplitude des vagues scélérates. Pourtant, dans les zones côtières, les conditions de profondeur infinie ne sont pas rencontrées. Il faut donc prendre en compte les effets bathymétriques, qui jouent un rôle sur le caractère dispersif du train d'onde. Paradoxalement, la focalisation de train de houle dans ces conditions est peu étudiée. Dans ce travail, nous nous attachons à mener cette étude dans des conditions de profondeur finie. Nous avons étudié, expérimentalement et numériquement la propagation d'un train de houle focalisant, avec et sans. L'effet du courant sur les caractéristiques de la focalisation est discuté en détails.

\section{Dispositif expérimental}

Les essais ont été réalisés dans un canal à houle d'une longueur de $18 \mathrm{~m}$ pour une section de $0.5 \mathrm{~m}$ de large et $0.6 \mathrm{~m}$ de haut. Le canal est doté d'un circuit fermé de courant d'eau qui peut être généré dans les deux sens avec un système de commande pour fournir un écoulement uniforme à surface libre avec une couche limite de 3 à $4 \mathrm{~cm}$ au fond du canal et un débit variable. Les ondes sont générées à l'aide d'un batteur placé à l'extrémité du canal contrôlé par un ordinateur qui excite des impulsions à différentes fréquences et amplitudes avec et sans courant.

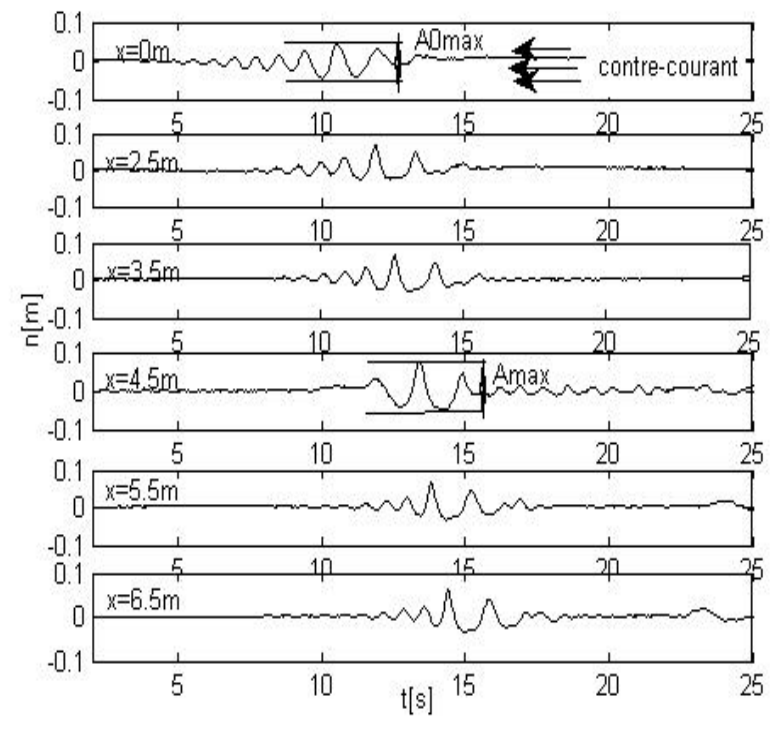

Figure 1. Evolution de la surface libre à contre courant $U=-0.08 \mathrm{~m} / \mathrm{s}, f=0.75 \mathrm{~Hz}$ à différentes positions le long du canal.

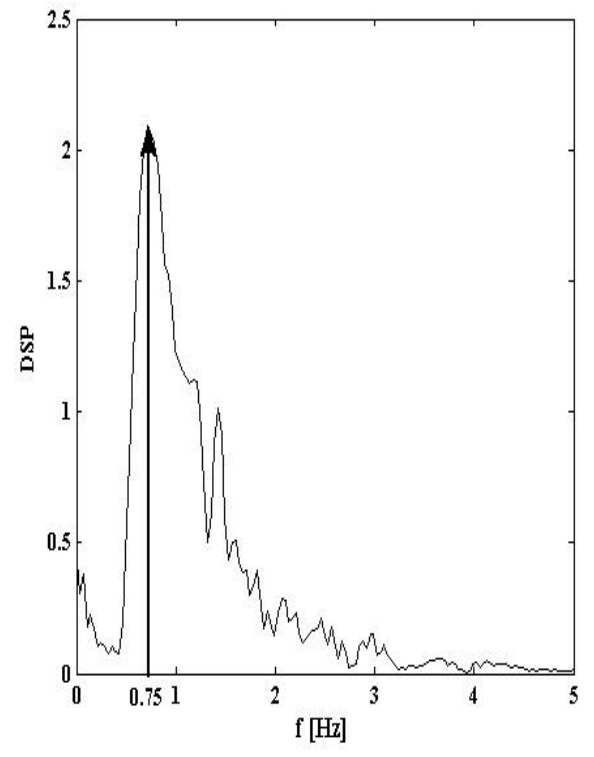

Figure 2. Densité spectrale d'une série temporelle de la surface libre présentée sur la figure 1. 


\section{XII ${ }^{\text {èmes }}$ Journées Nationales Génie Côtier - Génie Civil \\ Cherbourg, 12-14 juin 2012}

Dans ces expériences nous allons montrer l'influence des courants hydrodynamique sur les caractéristiques d'un paquet d'onde se propageant le long du canal. Pour cela, on procède à la mesure de l'évolution de la surface libre à l'aide de sondes résistives. La première sonde est maintenue dans une position fixe, à l'origine du canal, près du batteur. La seconde est déplacée le long du canal, pour plusieurs positions, dans une zone voisine du point de focalisation.

\section{Résultats}

\subsection{Etudes expérimentales}

En utilisant deux sondes résistives, nous avons effectué deux séries de mesures à deux fréquences modulées de pics $1.2 \mathrm{~Hz}$ et $0.75 \mathrm{~Hz}$, pour le cas de profondeur infinie, $f=1.2 \mathrm{~Hz}$ avec $h=32 \mathrm{~cm}$ (impulsion 1) et $f=0.75 \mathrm{~Hz}$ avec $h=25 \mathrm{~cm}$ (impulsion 2) pour le cas profondeur finie. Le but est de faire une comparaison et savoir comment le changement de la fréquence influence le comportement de la houle. La figure 1 où $k h$ est estimé sans courant, montre l'évolution de la surface libre pour les ondes focalisées en présence d'un courant hydrodynamique.

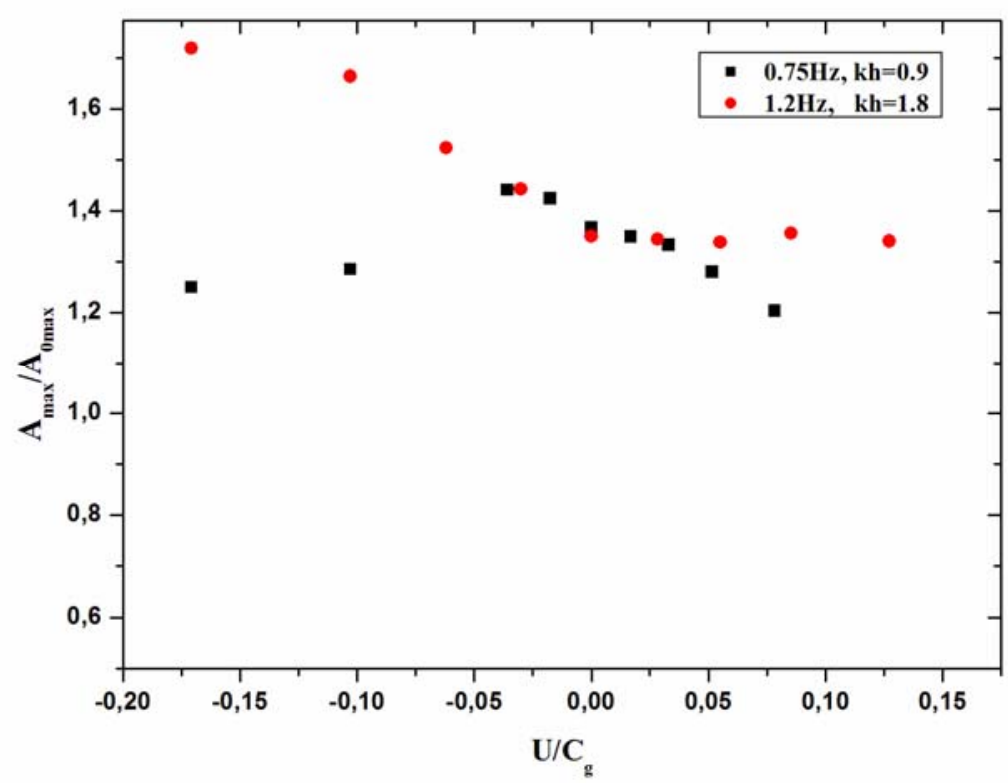

Figure 3. Variation de l'amplitude de focalisation pour $f=1.2$ et $0.75 \mathrm{~Hz}$.

Dans une expérience reproductible, le point de focalisation est défini comme l'endroit où l'élévation maximale de la surface libre est atteinte. D’après nos résultats expérimentaux nous avons constaté que le groupe qui se propage avec un courant positif se focalise plus tard que celui qui se propage sans courant. D'autre part le groupe qui se 
propage à contre courant (figure 1) focalise plus rapidement que celui qui se propage sans courant. Ainsi le déferlement peut intervenir d’une manière plus rapide dans le cas contre courant que pour le co-courant ou sans courant. Ces résultats sont en accord avec les expériences de WU \& YAO (2004) pour localiser la limite de déferlement en présence d'un courant.

On présente dans la figure 3 l'élévation maximale de la surface libre au point de focalisation en fonction de la vitesse du courant pour les deux cas de fréquence d'excitation ( $f=0,75 \mathrm{~Hz}$ et $1,2 \mathrm{~Hz}$ ). $A_{\max } / A_{0 \max }$ représente le rapport de l'amplitude maximale au point de focalisation sur l'amplitude maximale initiale près du batteur et $U / C_{g}$ correspond au rapport de la vitesse d'écoulement sur la vitesse de groupe. Sur la figure 3, ce facteur d'amplification peut atteindre jusqu'à $40 \%$ en l'absence de courant. Cette élévation est due à la focalisation à une certaine distance loin de la première sonde. Il est remarquable que pour les deux courbes de différentes fréquences, l'amplitude maximale de la surface libre évolue d'une manière différente. L'évolution de l'amplitude à la surface libre à contre-courant peut atteindre plus de $65 \%$ et $40 \%$ pour le cas co-courant par rapport à l'amplitude initiale pour le cas d'une fréquence de 1.2 Hz. Cette évolution est similaire à celle trouvée par des simulations numériques de TOUBOUL et al. (2007). En outre, pour le cas d'une fréquence de $0.75 \mathrm{~Hz}$, l'augmentation atteint 25\%. Cette variation d'amplitude pour les deux fréquences est expliquée par la différence entre la dispersion $\left(\partial c_{g}(k) / \partial k\right)$ de la vitesse de groupe pour deux impulsions de fréquences modulées. Cette dispersion est plus importante pour l'impulsion 1 que dans le cas pour l'impulsion 2 Ainsi, ce phénomène conduit à observer une amplitude plus élevée pour la fréquence de $1.2 \mathrm{~Hz}$ que pour la fréquence de $0.75 \mathrm{~Hz}$. On montre sur la figure 4 les différents points de focalisation en fonction du rapport vitesse du courant sur la vitesse de groupe $\left(U / C_{g}\right)$ pour les deux cas de fréquence de houles $(f=0,75 \mathrm{~Hz}$ et $1,2 \mathrm{~Hz}$ ). On remarque que l'évolution des points de focalisation suit la même allure que celle trouvée théoriquement par TOUBOUL et al. (2007) pour le cas en eau profonde. Cependant pour le cas où la fréquence d'excitation est égale à $0.75 \mathrm{~Hz}$, les points de focalisation apparaissent dans une zone plus étendue. En particulier, on peut observer pour le cas du contre-courant $U / C_{g} \in[-0,1,0]$, les points de focalisation s'étendent sur une zone moins large par rapport au cas du co-courant. Contrairement à ce qu'on a observé avec une fréquence de $0.75 \mathrm{~Hz}$, pour une fréquence de $1.2 \mathrm{~Hz}$, les points de focalisation restent localisés dans une zone très étroite. La distribution des points de focalisation peut s'expliquer par le fait qu'une augmentation de la fréquence d'excitation implique l'augmentation de dispersion de la vitesse de groupe, cette augmentation amène à une diminution de la distance de focalisation. En revanche, une diminution de la fréquence conduit a une augmentation de la distance de focalisation. 


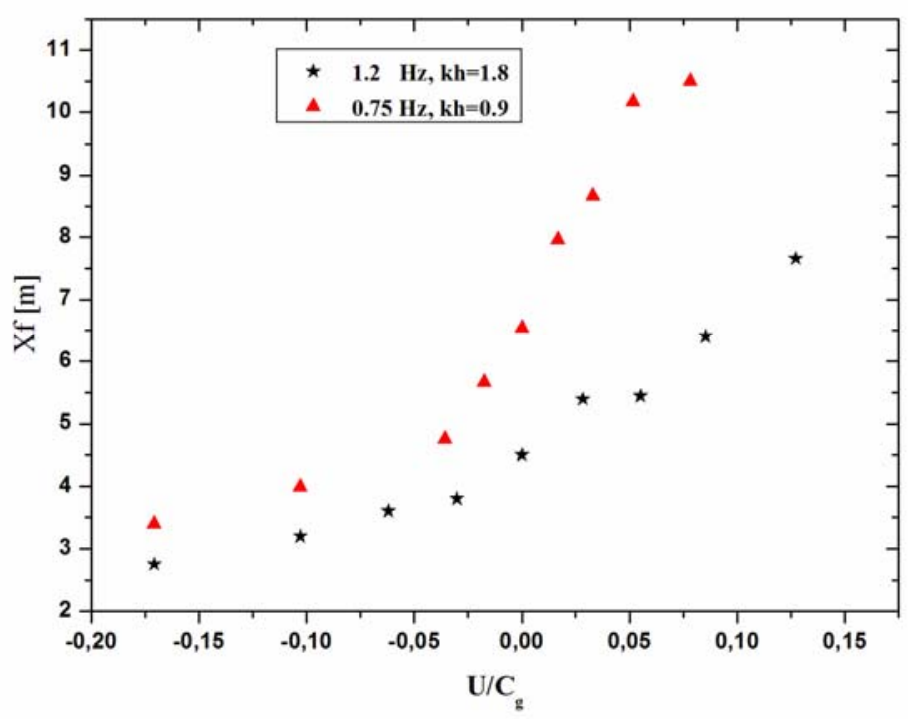

Figure 4. Variation de point de focalisation en fonction des vitesses du courant pour les deux fréquences $f=1.2$ et $0.75 \mathrm{~Hz}$.

Dans le but de donner une validation numérique à nos résultats expérimentaux, une série de simulations numériques d'un train d'ondes qui se propage avec ou sans courant dans une profondeur finie ont été réalisées. Le paquet d'ondes, avec modulation de fréquence linéaire est produit en un point fixe. La question est conçue comme un problème de conditions aux limites et non pas un problème de conditions initiales sans l’utilisation directe de la transformation de Galilée.

\subsection{Formulation mathématique du problème}

Mathématiquement, le problème des ondes se propageant à la surface d'un fluide se résout classiquement en théorie potentielle, en supposant que le fluide est non visqueux et incompressible. L'écoulement est incompressible et la vitesse du fluide dérive d'un potentiel des vitesses $\phi(x, z, t)$, qui satisfait à l'équation de Laplace $\Delta \phi=0$. Ainsi, le potentiel des vitesses peut être relié à l'élévation de la surface libre $\eta(x, t)$ grâce aux conditions aux limites non linéaires du problème. Ainsi, le problème complet correspond à l'équation de Laplace, considérée avec les conditions de fond et de surface libre suivantes :

$\varphi=\phi+U x$

$\Delta \phi=0$

$\frac{\partial \eta}{\partial t}+\frac{\partial \phi}{\partial x} \frac{\partial \eta}{\partial x}+U \frac{\partial \eta}{\partial x}=\frac{\partial \phi}{\partial z}$ and $\frac{\partial \phi}{\partial t}+U \frac{\partial \phi}{\partial x}+\frac{(\nabla \phi)^{2}}{2}+g \eta=0 \quad$ on $z=\eta(x, t)$

$\frac{\partial \phi}{\partial z}=0 \quad$ on $z=-h$. 
Une première approche permettant de résoudre le problème pourrait être obtenue en linéarisant les conditions cinématiques et dynamique de la surface libre. Dans ce cas, le problème considéré est purement linéaire, et admet les ondes d'Airy comme solution. Dans ce cas, la relation de dispersion linéaire $(\omega-k U)^{2}=g k \tanh (k h)$ doit être satisfaite. Ainsi, les composantes obtenues par transformée de Fourier d'une condition limite initiale appropriée peuvent être advectées indépendamment. Une transformée de Fourier inverse fournira alors l'évolution temporelle de l'élévation de la surface libre :

$$
\eta(x, t)=\frac{1}{2 \pi} \int_{-\infty}^{\infty}\left(\int_{0}^{\infty} \eta(0, \tau) e^{i \omega \tau} d \tau\right) e^{i(k x-\omega t)} d \omega
$$

Dans la suite, cette approche sera nommée le modèle linéaire. Une autre approche consiste à résoudre complètement le problème non linéaire pour $\phi$, tel que décrit par les équations (2-4). Cela peut être réalisé en utilisant la seconde identité de Green. Une description de type mixte Euler-Lagrange du problème est adoptée, ce qui signifie que l'on utilise une description particulaire de la surface libre. Plus de détails peuvent être trouvés dans TOUBOUL et al. (2007).

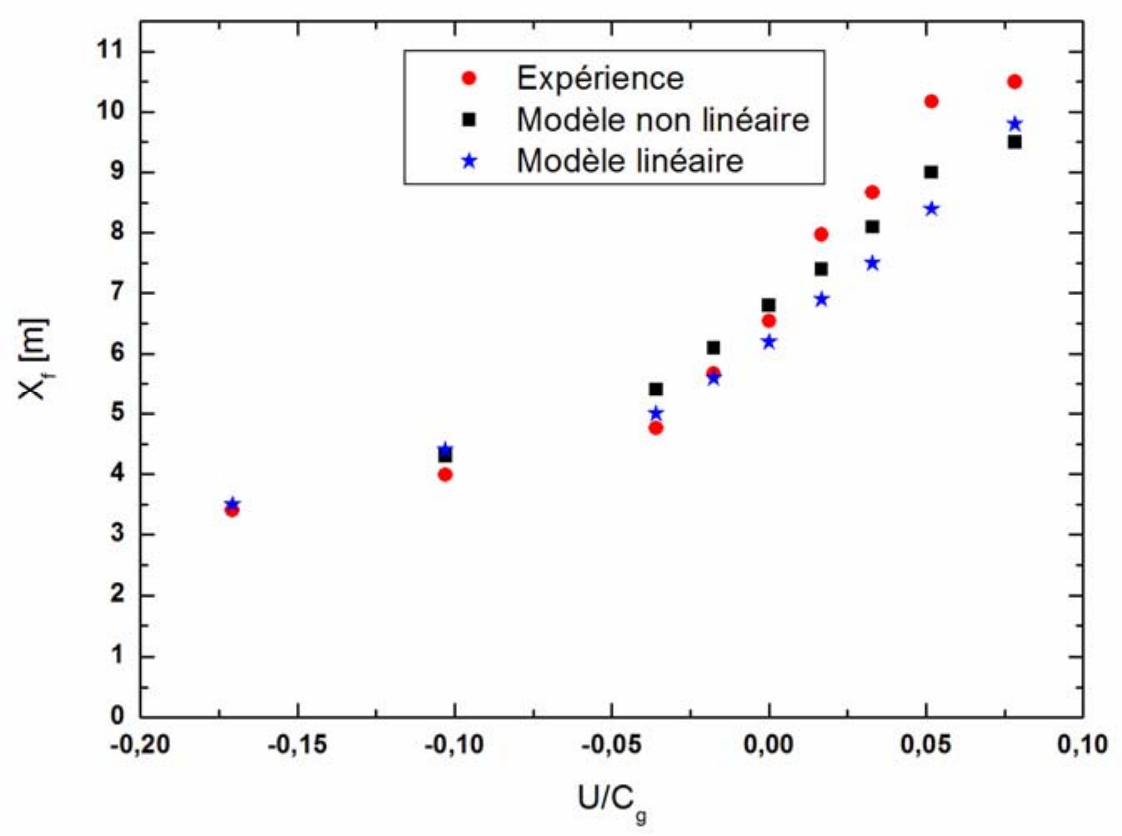

Figure 5. Comparaison de l'évolution du point de focalisation à une fréquence d'excitation moyenne $f=0.75 \mathrm{~Hz}$.

La figure 5 présente l'emplacement du point de focalisation obtenu à partir des méthodes à la fois linéaires et non linéaires qui sont comparés avec nos résultats expérimentaux, tracées en fonction de la vitesse du courant. On peut remarquer un très bon accord entre les méthodes linéaires et non linéaires avec les valeurs expérimentales. 


\section{XII ${ }^{\text {èmes }}$ Journées Nationales Génie Côtier - Génie Civil \\ Cherbourg, 12-14 juin 2012}

Des différences très faibles peuvent être observées entre les méthodes linéaires et non linéaires et les valeurs expérimentales, en particulier dans les contre-courants forts, où les vagues deviennent plus cambrées. Cela montre que d'un point de vue cinématique, la représentation linéaire du problème est une approche relativement pertinente.

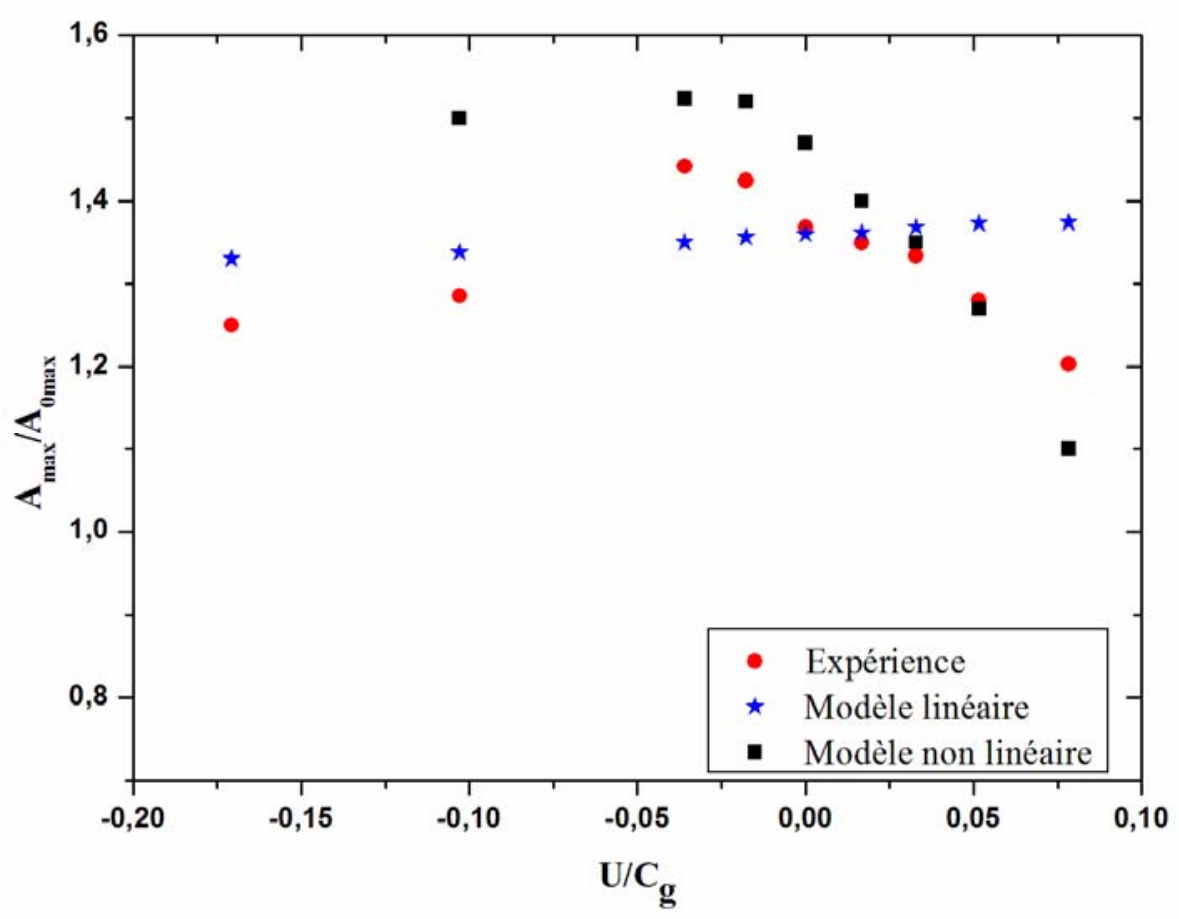

Figure 6. Comparaison des résultats numériques et expérimentaux de l'évolution de l'amplitude de focalisation, $f=0.75 \mathrm{~Hz}$.

La figure 6 montre l'amplitude maximale atteinte par les vagues en fonction de la vitesse du courant, pour les deux modèles linéaires et non linéaires. Les valeurs obtenues grâce au modèle linéaire sont légèrement différentes dans le cas où le maximum est atteint avec un co-courant. Ceci peut être compris, en considérant que le point de focalisation se transforme en une zone de focalisation dans les deux cas du contre et co-courant.

\section{Conclusion}

Nous avons constaté que la distance de focalisation dépend du sens du courant hydrodynamique. Cette longueur augmente pour une propagation dans le sens du courant et diminue en contre courant. Nous avons également montré que cette longueur décroît avec la diminution de rapport $H / \lambda$ (où $\lambda$ la longueur d'onde). En outre, la variation de l'élévation maximale de la surface libre au point de focalisation est fortement sensible aux changements de profondeur. Dans le cas d'eau de profondeur 
finie ( $f=0.75 \mathrm{~Hz}$ ), l'élévation de l'amplitude en présence d'un contre courant est moins importante par rapport au cas de profondeur infinie. Cela peut être expliqué par l'interaction des ondes superficielles avec l'écoulement cisaillé prés de fond en provoquant la diminution de l'amplitude des houles. Cette atténuation est due à la formation d'une couche limite turbulente conditionnée par le gradient de vitesse de contre courant généré dans le canal. D’autre part les simulations numériques ont été réalisées pour les équations du fluide non visqueux. On a constaté que les résultats expérimentaux sont en bonne concordance avec les simulations numériques, si le déferlement des ondes superficielles ne se produit pas suite à leur "focalisation".

\section{Références bibliographiques}

KHARIF C., PELINOVSKY E., TALIPOVA T., SLUNYAEV A. (2001). Focusing of Nonlinear Wave Groups in Deep Water. JETP Letters, Vol. 73, n 4, pp 170-175. doi:10.1134/1.1368708

KHARIF C., PELINOVSKY E. (2003). Physical mechanisms of the rogue wave phenomenon. European J. Mechanics B - Fluids, 22, pp 603-635. doi:10.1016/j.euromechflu.2003.09.002

PORUBOV A.V., TSUJI H., LAVRENOV I.V., OIKAWA M. (2005). Formation of the rogue wave due to nonlinear two-dimensional waves interaction, Wave Motion, Vol. 42, pp 202-210. doi:10.1016/j.wavemoti.2005.02.001

TOUBOUL J., PELINOVSKY E., KHARIF C. (2007). Nonlinear Focusing Wave Group on Current. Journal of Korean Society of Coastal and Ocean Engineers Vol. 19, $\mathrm{n}^{\circ}$ 3, pp 222-227.

WU C.H., YAO A. (2004). Laboratory measurements of limiting freak waves on currents, Journal of Geophysical Research, Vol. 109, C12002. doi:10.1029/2004JC002612 